\title{
Neue Darstellungsmethoden des Phenylesters der Eisentetranitrososulfosäure.
}

\author{
Von \\ K. A. Hofmane und O. F. Wiede. \\ (Mitteilung aus dem chem. Laboratorium der kgl. Akademie der Wissenseliaften \\ zu München.) \\ Dritte Mitteilung.
}

Wir haben vor kurzem eine neue Darstellungsweise von Estern der Eisentetranitrososulfosüure beschrieben. ${ }^{1}$ Es gelang uns durch Einleiten von Stickoxyd in ein wässeriges Gemisch von Eisenhydroxydul und Äthylmerkaptan den früher von $\mathrm{P}_{\Lambda \mathrm{VEL}}{ }^{2}$ durch Einwirkung von Jodäthyl auf das Kalisalz der Eisentetranitrososulfosäure dargestellten Ätbylester dieser Säure in befriedigender Ausbeute zu erhalten. Wiederholte Versuche ergaben schliefslich eine Maximalausbeute von $37 \mathrm{~g}$ aus $25 \mathrm{~g}$ Ester Merkaptan und $225 \mathrm{~g}$ Eisenvitriol. In analoger Weise vermochten wir das bis dahin noch unbekannte Dinitrosoeisenphenylmerkaptid aus seinen Komponenten aufzubauen. Die Bildung von Phenyldisulfid verminderte hier die Ausbeute derart, dafs wir nur im stande waren, den Schmelzpunkt $\left(179^{\circ}\right)$ und die Zusammensetzung durch eine vollständige Analyse festzustellen.

In letzter Zeit fanden wir nun, dals bei der Einwirkung sowohl von Phenylhydrazin, als auch von Diazobenzolnitrat auf das Kalisalz de: Eisenheptanitrososulfosäure der Phenylester der Tetrasäure $\mathrm{C}_{6} \mathrm{H}_{5} \mathrm{SFe}(\mathrm{NO})_{2}$ auf verhältrismälsig glatte Weise entsteht. - Mischt man reines Phenylhydrazin mit dem erwähnten trockenen Kalisalz $\mathrm{Fe}_{4}(\mathrm{NO})_{7} \mathrm{~S}_{3} \mathrm{~K}+\mathrm{H}_{2} \mathrm{O}$, so findet eine sehr lebhafte Reaktion statt Unter heftigem Aufschäumen entweicht Stickstoff und tritt ein intensiver Geruch nach Thiophenol auf, das bei der hohen Temperatur verdampft. Im Rückstande verbleibt hauptsächlich Eisenoxyd neben undefinierbaren Harzen. Um die Reaktion zu mälsigen wirkten wir in der Folge mit berechneten Mengen Phenylhydrazin (7 Mol.) einmal auf die wässerige, dann auch auf die alkoholische

1 Diese Leitschr. 9, 300.

- Ber. deutsch. chem. Ges. (1882), 2607. 
Lösung des Heptasalzes (1 Mol.) ein. Trotz der Verdünnung durch die angewandten Lösungsmittel ist es zweckmälsig, unter stetiger Liskühlung zu arbeiten. Alsbald nach Zugabe des Phenylhydrazins tritt schwache Gasentwickelung ein. Nach ungefähr 2 Tagen ist die Reaktion beendet und an Stelle des charakteristischen Kaliumheptasulfonates krystallisieren aus der alkalisch reagierenden Flüssigkeit braune glänzende Blättchen aus, vermischt mit braunem, amorphen Eisenoxyd. Man nimmt in wenig heifsem Benzol auf, filtriert und lälst durch Abkühlen krystallisieren. Das entstehende krystallinische Produkt zeigt den Schmelzpunkt 179 . Die Kontrolle für die Reinheit lälst sich übrigens hier durch den Schmelzpunkt nicht mit der gewohnten Sicherheit führen. Es zeigt sich nämlich, dafs heifser Alkohol oder Aceton geringe Mengen von Phenyldisulfid (Schmelzpunkt $60^{\circ}$ ) ausziehen, trotzdem die Schärfe des Schmelzpunktes keine Beimengung verriet. Man erhält infolge dessen leicht ein gewisses Plus an Kohlenstoff. Über Schwefelsäure im Vakuum 1 Stunde getrocknet ergab die Substanz bei der Analyse die Formel $\mathrm{C}_{6} \mathrm{H}_{6} \mathrm{SFe}\left(\mathrm{NO}_{2}\right)$ :

\begin{tabular}{c|c|c}
\hline & Gefundene Prozente & $\begin{array}{c}\text { Berechnete } \\
\text { Prozente }\end{array}$ \\
\hline Fe & 25.3 & 24.9 \\
$\mathrm{~N}$ & 12.5 & 12.4 \\
$\mathrm{~S}$ & - & 14.2 \\
$\mathrm{C}$ & $33.3,33.3,33.5$ & 32.0 \\
$\mathrm{H}$ & $2.38,2.3,2.4$ & 2.2
\end{tabular}

Zur Eisenbestimmung wurde die Verbindung mit rauchender Salpetersäure zersetzt und das Eisen mit Ammoniak zur Fällung gebracht. Beim einfachen Glühen der Substanz ist dieselbe zu flüchtig, und gehen daher Eisenoxydflocken in grofser Menge fort. Die Stickstoffbestimmung, sowie die Kohlenstoffbestimmung geschah durch Verbrennen im Rohr. Bei der Kohlenstoffbestimmung war das Kupferoxyd mit grobkörnigem Bleichromat gemischt, um etwaige Sauerstoffverbindungen des Schwefels zurückzuhalten. Um das Übergehen höherer Stickoxyde zu vermeiden, waren 2 gut reduzierte Kupferspiralen vorgelegt. Aufserdem war die Substanz im Schiffchen innig mit Bleichromat gemengt. - Auffallend war, dafs zu den Verbrennungen kein Rohr zweimal benutzt werden konnte, da nach jeder Verbrennung beim Erkalten dasselbe an der Stelle des SchiffZ. anorg. Chem. XI. 
chens zersprang. Es ist dies wohl auf eine teilweise Flüchtigkeit der Verbindung zurückzuführen, wie man denn auch jedesmal einen Anflug von Eisenoxyd an den Glaswandungen beobachten konnte.

Es liegt in obiger Verbindung also der Phenylester der Tetrasäure vor, trotzdem sich dieses aus Alkohol erhaltene Präparat durch sein Aussehen, braune Krystallplättchen, unterschied von den aus wässeriger Lösung erhaltenen schwarzen blätterigen Krystallen. ${ }^{1}$ Es kann also der Phenylester auch im reinen Zustande je nach der Darstellungsweise glänzend braune, oder glänzend schwarze Plättchen bilden, die aber den gleichen Schmelzpunkt besitzen, auch hinsichtlich ihrer Löslichkeit in Benzol keinen Zweifel an der Identität zulassen. Wahrscheinlich hängt die Verschiedenheit der Färbung mit dem ausgeprägten Dichtroismus, der der ganzen Klasse der Nitrosoeisenverbindungen eigen ist, zusammen.

Die Reaktion des Phenylhydrazins mufste zunächst befremden, doch fanden wir bald eine befriedigende Aufklärung ihres Verlaufes.

Bringt man nämlich Diazobenzolnitrat in äquimolekularer Menge zu dem Kalisalz der Eisenheptanitrososulfosäure, so entsteht in guter Ausbeute der mehrfach erwähnte Phenylester. $12 \mathrm{~g}$ reines krystallisiertes Diazobenzolnitrat werden allmählich zu der berechneten Menge Kaliumeisenheptasulfonat in abs. Alkohol hinzugefügt. Trotz guter Eiskühlung findet alsbald Gasentwickelung statt. Es entweicht Stickstoff und nach wenigen Minuten fällt ein Gemenge von Eisenoxyd und dem krystallisierten Nitrosoeisenphenylmerkaptid aus. Der Niederschlag wird mit heifsem Alkohol extrahiert und dann in siedend heifsem Benzol gelöst. Beim Erkalten krystallisierten braune, glänzende Plättchen aus, die bei $179^{\circ}$ schmolzen und, über Schwefelsäure während einer Stunde im Vakuum getrocknet, bei der Analyse $25.4 \% \mathrm{Fe}$ ergaben. Für $\mathrm{C}_{6} \mathrm{H}_{5} \mathrm{SFe}(\mathrm{NO})_{2}$ folgen $24.9 \% \mathrm{Fe}$.

Aus dem Kalisalze der Heptasäure ist also durch Einwirkung von Diazobenzolnitrat der Phenylester der Tetrasäure entstanden. Nun hat Leuckakr' ${ }^{2}$ eine interessante Darstellungsweise von Thiophenolen gefunden, die darauf beruht, dafs aus Diazobenzolchlorid und xanthogensaurem Kali unter Stickstoffentwickelung der Xanthogensäurephenylester gebildet wird, der dann beim Kochen mit alkoholischem Kali in Kohlenoxysulfid und in Thiophenol sich spalten lärst.

1 Diese Zeitschr. 9, 302.

${ }^{2}$ Journ. pr. Ohem. (1890) 41, 186. 
Mit Vorteil lärst sich das xanthogensaure Kali ersetzen ${ }^{1}$ durch das aus Schwefellkohlenstoff und Kali hergestellte Dithiokarbonat. Dies liefert mit Diazobenzolchlorid $\mathrm{CO}\left(\mathrm{SC}_{6} \mathrm{H}_{5}\right)_{2}$ und dies beim Verseifen Thiophenol. Aus Thiosulfaten und Diazobenzolsalzen entstehen ölige Produkte, die beim Verseifen in Thiophenol und Sulfite zerfallen. Aus diesen Beispielen geht hervor, dals an Schwefel gebundenes Kalium bei der Einwirkung von Diazobenzolchlorid gegen den Phenylrest unter Stickstoffabspaltung ersetzbar ist.

In unserem Falle sollte aus Kaliumheptasulfonat und Diazobenzolsalz der Phenylester der Heptasäure entstehen. Nun hat aber bereits PAver gefunden, und wir konnten diese Beobachtung bestätigen, dafs die Salze der Heptasäure durch Jodäthyl zersetzt werden. Wirkt man mit Alkali auf das Heptasalz ein, so entsteht daraus Tetrasalz und dies giebt mit Jodäthyl leicht den Äthylester. Es scheint, als ob der Aufbau des Heptasäuremoleküls den Eintritt umfangreicherer Reste an Stelle des Säurewasserstoffs nur unter gleichzeitiger Sprengung des Moleküls zulielse. Da nun bei der von Pavel studierten Aufspaltung der Heptasäure durch Einwirkung von Alkali Tetrasalz entsteht, so dürfte die Bildung eines Phenylesters der Tetrasäure bei Verwendung von Diazobenzolnitrat wohl erklärt sein.

Dafs Phenylhydrazin bei Gegenwart der zahlreichen Nitrosogruppen der Heptasäure vorübergehend in Diazobenzol, wohl durch einen Oxydationsvorgang auf Kosten eines Teiles der Heptasäure, verwandelt werden kann, erscheint uns möglich, und so wäre ein Zusammenhang zwischen den beiden eben beschriebenen Bildungsweisen des Dinitrosoeisenphenylmerkaptids gefunden. Zum Schlusse möchten wir noch die Molekulargewichtsbestimmung unseres nunmehr leicht erhältlichen Körpers anfügen. Zwar löst sich unser Ester in kaltem Benzol nur schwer, doch konnten wir zu befriedigenden Resultaten gelangen:

\begin{tabular}{|c|c|c|}
\hline Lösungsmittel & $\begin{array}{l}\text { Angewandte } \\
\text { Substanz }\end{array}$ & Depression \\
\hline $29.43 \mathrm{~g} \mid \mathrm{x}_{1}$ & 0.0714 & $0.029^{\circ}$ \\
\hline Benzol $\mathrm{x}_{2}$ & 0.1626 & $0.068^{\circ}$ \\
\hline
\end{tabular}

'Lustia, Chem. Ber. (1891), 565 R. Daccomo, Centralbl. (1891) 2, 657. 
Eine nochmalige Bestimmung mit sorgfältigst gereinigtem Material ergab:

\begin{tabular}{|c|c|c|}
\hline Lösungsmittel & $\begin{array}{c}\text { Angewandte } \\
\text { Substanz }\end{array}$ & Depression \\
\hline $23.33 \mathrm{~g} \int \mathrm{x}_{1}$ & 0.2893 & $0.147^{\circ}$ \\
\hline Benzol & 0.3185 & $0.162^{0}$ \\
\hline
\end{tabular}

Molekulargewicht: $\mathrm{x}_{1}=422, \mathrm{x}_{2}=420$.

Berechnetes Molekulargewicht für $\left[\mathrm{C}_{6} \mathrm{H}_{5} \mathrm{SFe}(\mathrm{NO})_{2}\right]_{2}=450$.

Also auch dieser Ester besitzt wie der Athylester ${ }^{1}$ in Benzollösung ein doppelt so grofses Molekül, als wir in unseren Analysenformeln angeben. Indessen haben die Doppelformeln, soweit es sich um die bis jetzt bekannten Reaktionen dieser Körper, wie auch der Tetrasäure selber handelt, keinen Vorteil vor den einfachen voraus.

1 Diese Zeitschr. 9, 299.

Bei der Redaktion eingegangen am 5. Dezember 1895. 